those who continued to receive tamoxifen, patients who switched to anastrozole had significantly higher disease-free survival (hazard ratio $[\mathrm{HR}] 0.59 ; P<0.0001)$, event-free survival (HR 0.55; $P<0.0001$ ) and distantrecurrence-free survival (HR 0.61; $P=0.0015$ ). The benefit of switching to anastrozole was independent of nodal or receptor status, previous chemotherapy and tumor size.

These findings validate the ASCO treatment guidelines for women with hormone-sensitive early-stage breast cancer, which favor the use of an aromatase inhibitor following initial tamoxifen therapy, and challenge the current standard approach of 5 years of adjuvant tamoxifen.

Original article Jonat W et al. (2006) Effectiveness of switching from adjuvant tamoxifen to anastrozole in postmenopausal women with hormone-sensitive early-stage breast cancer: a meta-analysis. Lancet Oncol 7: 991-996

\section{Reducing the time between doses is feasible in breast cancer chemotherapy}

A recent clinical trial (CALGB 9741) has indicated that the administration of chemotherapy every 14 days, as opposed to every 21 days, is beneficial for both disease-free and overall survival. As a result, Fornier et al. have carried out a pilot study of 38 women with early-stage breast carcinoma to assess the feasibility of further reducing the interdose period to 10-11 days.

Patients were treated with 4 cycles of $100 \mathrm{mg} / \mathrm{m}^{2}$ epirubicin and $600 \mathrm{mg} / \mathrm{m}^{2}$ cyclophosphamide followed by 4 cycles of $175 \mathrm{mg} / \mathrm{m}^{2}$ paclitaxel. The majority of doses (81\%) were administered at intervals of no more than 1011 days (median interval 10 days). All patients received daily filgrastim between doses, delivered subcutaneously at $5 \mu \mathrm{g} / \mathrm{kg}$ body weight. Tolerability was defined as absence of any grade 3 or higher nonhematologic toxicity (excluding possible effects of filgrastim); $76 \%$ of patients achieved this criterion. After a median follow-up of 21.3 months, disease-free and overall survival were both $100 \%$ and there was no treatment-related long-term toxicity.

Fornier et al. documented a high incidence of febrile neutropenia in their study relative to the CALGB 9741 trial (16\% vs 2\%), as well as a high hospitalization rate. They conclude that 10-11-day intervals between doses are feasible for treatment of breast cancer, but that use of the shorter cycle could be justified only after comparison with a 14-day regimen in a phase III clinical trial.

Original article Fornier MN et al. (2007) Increased dose density is feasible: a pilot study of adjuvant epirubicin and cyclophosphamide followed by paclitaxel, at 10- or 11-day intervals with filgrastim support in women with breast cancer. Clin Cancer Res 13:223-227

\section{Correlation of blood vessel type and prognosis in patients with CCRCC}

Recent studies have suggested that increased intratumoral microvascular density (MVD) correlates with poor prognosis in various malignancies, such as breast, lung, nasopharyngeal and prostate cancer; however, its association with clear-cell renal cell carcinoma (CCRCC) has been controversial. A new study by Yao et al. has evaluated the relationship between tumor vasculature in CCRCC and patient survival.

The study included specimens from 78 patients who were diagnosed with CCRCC and underwent nephrectomy between 1983 and 2004. The authors identified two types of microvessels: undifferentiated vessels that were $\mathrm{CD} 31^{+} / \mathrm{CD} 34^{-}$with small or absent lumen, thick walls, and no pericyte coverage; and differentiated vessels that were CD34 ${ }^{+}$. Tissue microarrays were prepared from all 78 samples. Higher differentiated MVD was correlated with lower tumor grade and longer patient survival, while higher undifferentiated MVD was correlated with higher pathologic grades and shorter survival. In fact, a high density of undifferentiated microvessels was an independent prognostic indicator of shorter survival. Patients with low undifferentiated and high differentiated MVD had a significantly longer survival time than did patients with high undifferentiated and low differentiated MVD $(P=0.0029)$.

On the basis of these results, a high differentiated MVD seems to indicate a better prognosis. The authors suggest that undifferentiated blood vessels might have potential as future therapeutic targets.

Original article Yao X et al. (2007) Two distinct types of blood vessels in clear cell renal cell carcinoma have contrasting prognostic implications. Clin Cancer Res 13: 161-169 